\title{
Synthesis and Characterization of Peptide-Chitosan
}

\section{Conjugates (PepChis) with Lipid Bilayer Affinity and}

\section{Antibacterial Activity}

Thais H. Costa Petrin,,$^{\dagger}$ Valmir Fadel, ${ }^{\S}$ Danubia B. Martins, ${ }^{\S}$ Susana A. Dias, ${ }^{,}$Ana Cruz, ${ }^{\star}$ Luciana Marciano Sergio, ${ }^{\perp}$ Manoel Arcisio-Miranda,${ }^{\perp}$ Miguel Augusto R. B. Castanho, $¥$ Marcia P. dos Santos Cabrera $^{*}, \xi \xi$

†Departamento de Química e Ciências Ambientais and ${ }^{\S}$ Departamento de Física, Universidade Estadual Paulista (Unesp), Instituto de Biociências Letras e Ciências Exatas (Ibilce), Câmpus São José do Rio Preto, SP, Brazil.

† Instituto de Medicina Molecular, Faculdade de Medicina, Universidade de Lisboa, Lisbon, Portugal.

${ }^{\perp}$ Laboratório de Neurobiologia Estrutural e Funcional (LaNEF), Departamento de Biofísica, Universidade Federal de São Paulo, São Paulo, Brasil. 


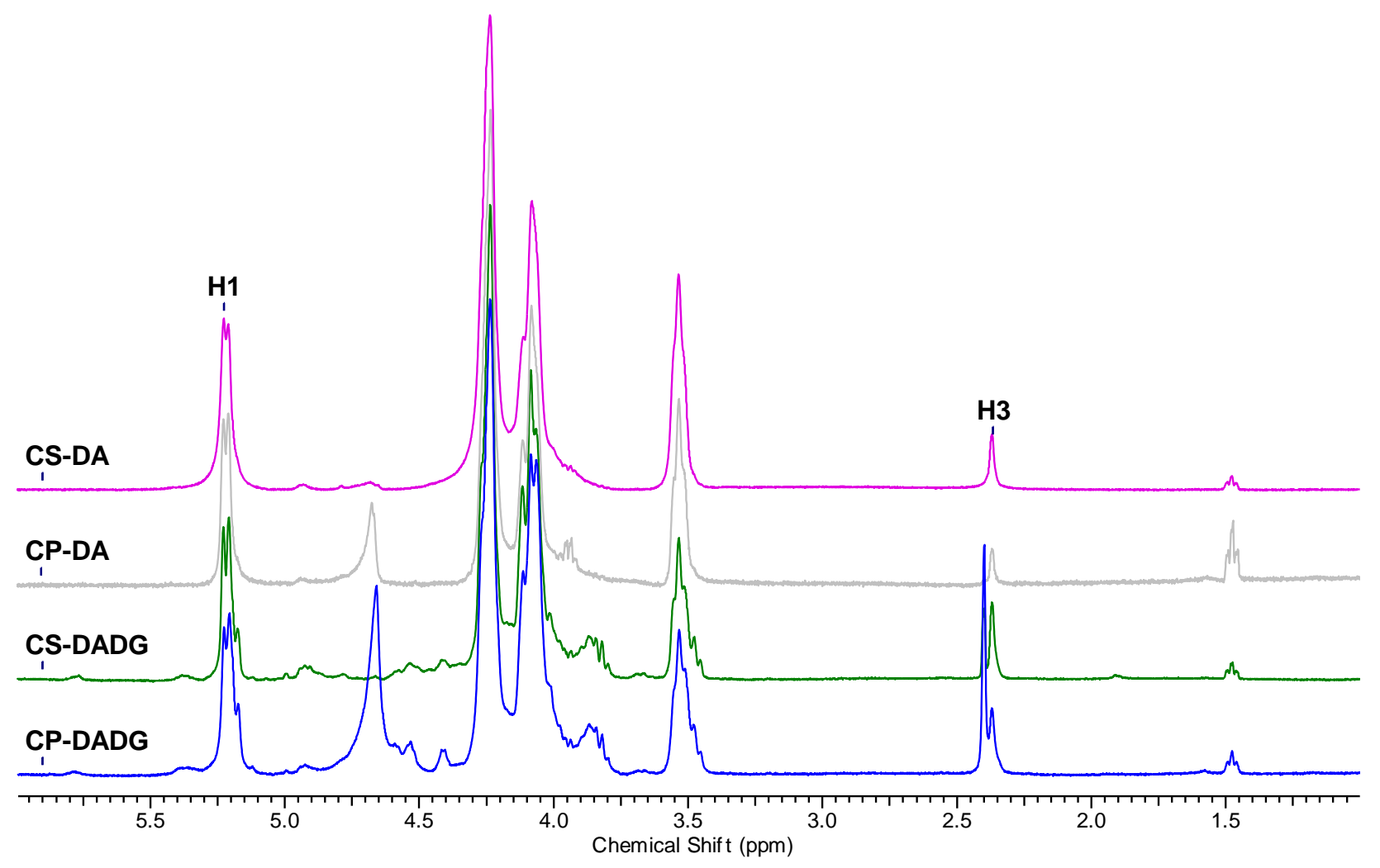

Figure SI1. ${ }^{1}$ H-NMR spectra of chitosans after deacetylation and subsequent degradation processes. CSDA and CP-DA are reagent and commercial grade chitosan samples after deacetylation, respectively. CSDADG and CP-DADG are the same deacetylated chitosan after the degradation process. The indicated $\mathrm{H} 1$ and $\mathrm{H} 3$ peaks correspond, respectively, to ${ }^{1} \mathrm{H}$ resonances of hydrogen bound to the anomeric carbon $(5.22 \mathrm{ppm})$ and hydrogen from the methyl group of the acetylglucosamine unit (2.37 ppm). 

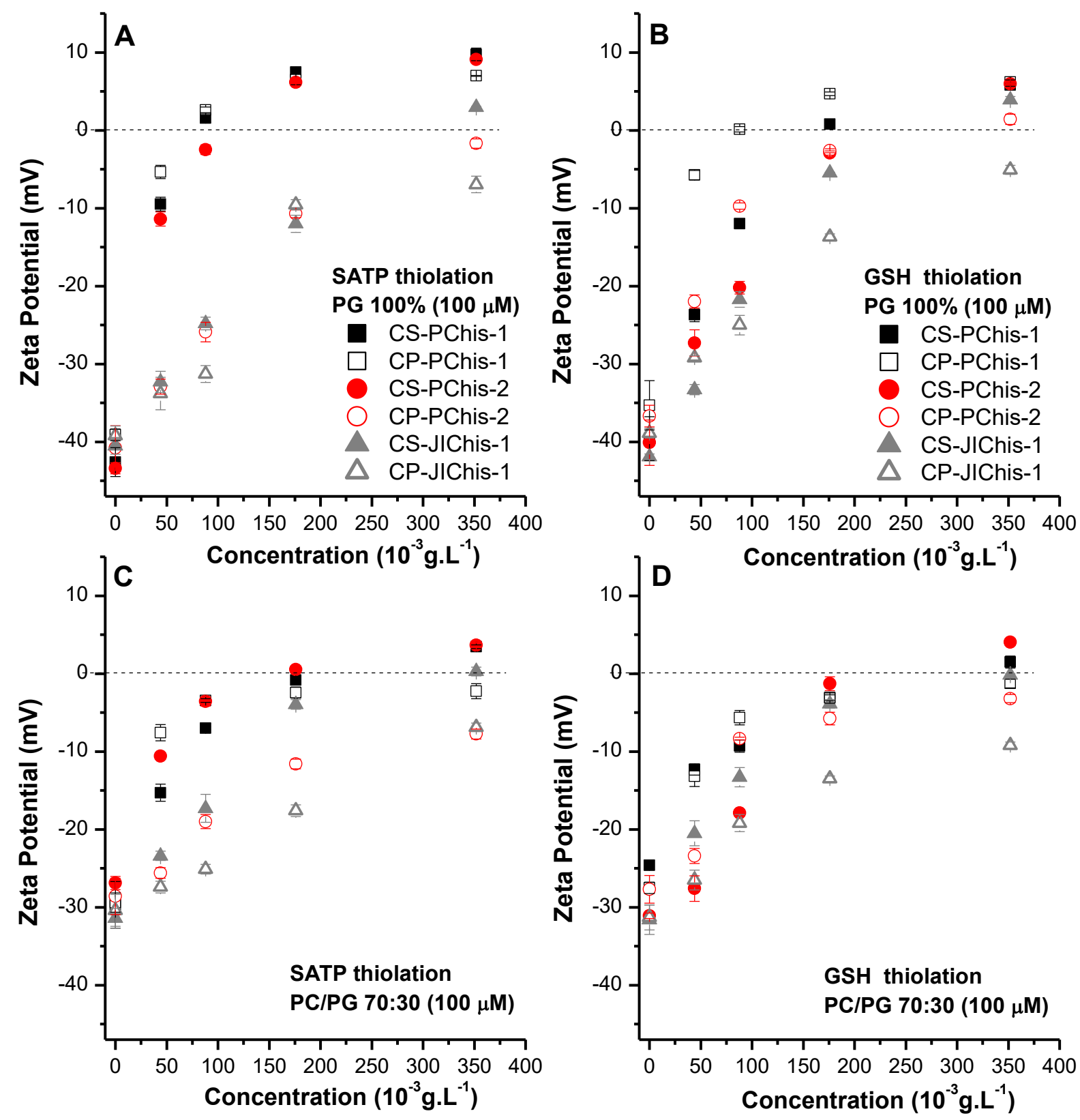

Figure SI2. Charge neutralization obtained in zeta potential determinations for PepChis conjugates in the presence of $100 \mu \mathrm{M}$ LUVs made of PG $100 \%$ or PC/PG $70: 30$, at $\mathrm{pH} 5.5$ and $25{ }^{\circ} \mathrm{C}$. A) and C) for conjugates obtained via SATP. B) and D) for conjugates obtained via GSH. Dashed line indicates the point of charge reversal. Closed symbols for CS- and open symbols for CP-derivatives. Error bars are about the size of symbols. 
Table SI1. Representative DLS determination of vesicles diameters in the absence $\left(\varnothing_{\mathrm{i}}\right)$ and in the presence of $88 \times 10^{-3}$ g.L.- PepChis conjugates.

\begin{tabular}{|c|c|c|c|c|}
\hline \multirow{2}{*}{$\begin{array}{c}\text { PepChis } \\
\text { conjugates } \\
\text { at } 88 \times 10^{-3} \text { g. } \mathrm{L}^{-1}\end{array}$} & \multicolumn{2}{|c|}{$\begin{array}{c}\text { PG } 100 \% \\
\emptyset_{\mathrm{i}}=126 \pm 1 \mathrm{~nm}\end{array}$} & \multicolumn{2}{|c|}{$\begin{array}{c}\text { PC/PG 70:30 } \\
\varnothing_{\mathrm{i}}=133 \pm 1 \mathrm{~nm}\end{array}$} \\
\hline & $\varnothing_{\mathrm{i}}(\mathrm{nm})$ & \pm s.d. & $\varnothing_{\mathrm{i}}(\mathrm{nm})$ & \pm s.d. \\
\hline CSsatp-PChis-1 & 3470 & 677 & - & - \\
\hline CPsatP -PChis-1 & 3054 & 1063 & 2594 & 599 \\
\hline CSsatP-PChis-2 & 3169 & 1112 & 2499 & 688 \\
\hline CPsatP-PChis-2 & 2126 & 366 & 4140 & 167 \\
\hline CPsatP-JIChis-1 & 2818 & 604 & 3568 & 121 \\
\hline CSsATP-JIChis-1 & - & - & 3618 & 510 \\
\hline CSgSh-PChis-1 & 5787 & 825 & 3367 & 854 \\
\hline CPGSh-PChis-1 & 5237 & 1337 & 6132 & 636 \\
\hline $\mathrm{CS}_{\text {gSh-PChis-2 }}$ & 3047 & 725 & 3640 & 2 \\
\hline CPGSh-PChis-2 & 3800 & 92 & 1654 & 296 \\
\hline CSgSh-JIChis-1 & 2311 & 719 & 4870 & 484 \\
\hline CPGSH-JIChis-1 & 1797 & 351 & 2083 & 1161 \\
\hline
\end{tabular}

